\title{
Single station lightning location using azimuth and time of arrival of sferics
}

\author{
Zahra Koochak $^{1}$, Antony Fraser-Smith ${ }^{1,2}$ \\ ${ }^{1}$ Department of Electrical Engineering, Stanford University, California, USA. \\ ${ }^{2}$ Department of Geophysics, Stanford University, California, California, USA.
}

Key Points:

- A novel method to define emission time and location of lightning

- Less source-intensive and widely applicable

- Improved and employed magnetic direction finding method for single station 


\begin{abstract}
Lightning strikes produce electromagnetic waves, now referred to as sferics, in the very low frequency (VLF, 3-30 kHz) and the extremely low frequency (ELF, 3-3k Hz) bands. Within these frequency bands, the Earth and ionosphere form a waveguide in which sferics propagate long distances with low attenuation. The structure of the received sferic waveform is mainly a function of propagation distance and the waveguide's parameters. This suggests that each observed sferic waveform contains information about the distance that this sferic has propagated which can be used to geolocate lightning. There are various approaches for analyzing received sferics, which mostly rely on measurements from multiple stations. However, in these methods, each station imposes an additional cost for building, maintenance, and synchronization. Here we present a novel method to estimate both the emission time and location of lightning, which works by measuring sferics recorded at a single station. We first process the sferic waveforms to obtain the arrival times of the VLF and ELF radiation components which propagate with different speeds. Once these two separate arrival times are determined, we use them to approximate the distance the sferic propagated in the Earth-ionosphere waveguide. We have used this novel method in combination with a method to find sferic direction to geolocate a significant number of lightning strikes for July 4, 2013. Using this proposed method, the distance of propagation estimates are accurate to within $6.7 \%$ of the NLDN determined propagation distance and the direction of propagation estimates are accurate to within $\sim 1.3 \%$ of the NLDN determined direction.
\end{abstract}

\title{
1 Introduction
}

Electromagnetic waves originating from lightning are called radio atmospherics, or sferics. The VLF and ELF components of the sferics propagate global distances through the Earth-ionosphere waveguide.A number of studies have used these components individually or together to geolocate lightning from recorded sferic data(Said,R. et al., 2010; Price, C. et al., 2002). Figure 1 shows the VLF and ELF components of a typical sferic waveform. The VLF component, highlighted in pink on the left, is the initial burst, which is followed by the ELF component, highlighted on the right. In Figure 1, the magnitudes of both components are normalized (the plotted magnitude is $\frac{M-\operatorname{mean}(M)}{\max (M)}$, where $\mathrm{M}$ is the measured time-domain signal amplitude). The VLF and ELF components of different sferics vary in time duration, but in general the VLF component is considerably shorter than 


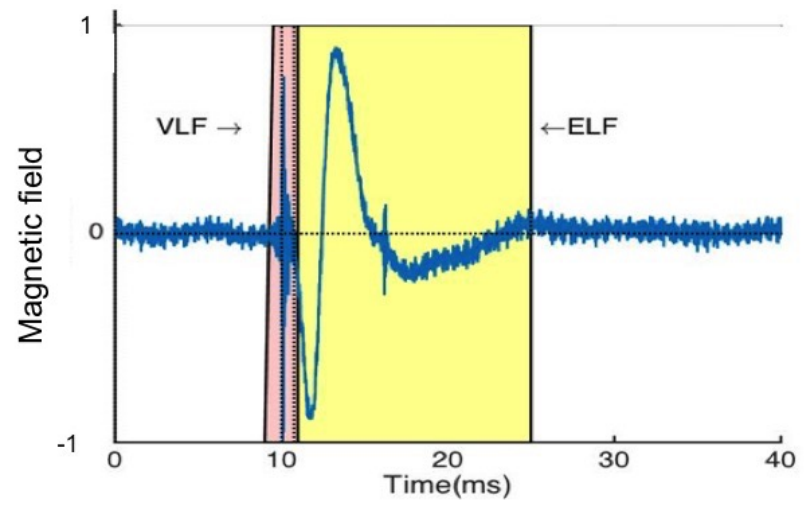

Figure 1. An example of a sferic waveform and the two associated VLF and ELF and components (recorded at Arrival Height on June 6, 2013 at 06:30 A.M.)

the ELF component. The data set used in this paper were recorded at two stations, one located at Arrival Height, Antarctica $\left(78^{\circ} \mathrm{S}, 167^{\circ} \mathrm{E}\right)$, and the other at Sondrestrom, Greenland $\left(66.99^{\circ} \mathrm{N}, 50.95^{\circ} \mathrm{W}\right)$, on June 6th and July 4th, 2013, respectively. In each of the stations, sferics were measured by two orthogonal magnetic loop antennas in North-South (NS) and East-West (EW) geomagnetic directions. The data are recorded with a sampling frequency of $100 \mathrm{kHz}$, and antennas are capable of measuring horizontal magnetic field in frequencies ranging from $300 \mathrm{~Hz}$ to $40 \mathrm{kHz}$.

Using the above data and our proposed method, we first estimated the distances of the lightning strikes and then compared the accuracy of our estimations with the locations estimated by the National Lightning Detection Network's (NLDN) multi-station method.

\section{Current Lightning Location Methods}

Several prior studies have attempted to utilize observed sferics at multiple ground stations to locate lightning. Some of them are based on multi station detections and use a central processor to employ Time of Arrival (TOA) and Magnetic direction finding (MDF) techniques (Nag et al., 2015, Said, R., 2017). NLDN and the Global Lightning Detection Network (GLD360) are examples of multi-station networks used to locate lightning. NLDN, operated by Vaisala, Inc., employs a network of low Frequency (LF) and VLF sensors to provide lightning location within the continental United States. Using NLDN, the arrival time and azimuth are measured with accuracies of $1.5 \mu \mathrm{s}$ and $1^{\circ}$, respectively (Cummins \& Murphy, 2009). The detection efficiency is estimated to be $\sim 60-76 \%$ for 
cloud to ground strokes. The median location error, as an important evaluation metric (Murphy, M, 2018), was reported around $308 \mathrm{~m}$ (Nag et al., 2011). In GLD360, each station correlates observed sferic data with a waveform-bank. Multiple stations then send their results to a central processor to approximate the lightning's time and locations. This method achieves $57 \%$ flash detection efficiency and $\sim 2-5 \mathrm{~km}$ accuracy (Said,R et al.; 2010; Said,R et al., 2013 ).

Alternative methods exist to locate lightning using mostly VLF measurements from a single station. For example in (Byerley, III et al., 1991) the ratio between radiation field component (Er) and an electrostatic field component (Es) is used for close-range lightning detection. The ratio of electric and magnetic fields or wave impedance also have been used to estimate propagation distance using a single station (Burke et al., 1995). However, these methods require the availability of both electric and magnetic field antennas, introducing additional cost and complexity in each station, which also is a concern in multistation locating methods. Ramachandran, V, et al. ,2007 described another method which works based on lightning-generated VLF sferics received in a single station. The reports $8.8 \%$ average distance estimation error, but it doesn't benefit from using ELF components of the sferic to improve distance estimations.

On the other hand, Wait developed a theory proposing that lightning can be located by examining the structure of recorded sferic data from a single location. Wait, J. R., 1960 also proposed a theory to estimate propagation distance by modeling the ELF component of the sferics.

However, Wait's method suffers from limited accuracy. An average error of $45.1 \%$ for daytime conditions with a standard deviation of $13.8 \%$ is reported for his method (Mackay, C.E.J et al., 2010). Wait assumed a certain analytical form for the lightning current moment, which non-ideally can be used to model the ELF component of the sferic. Later more accurate approximation for source current moment was introduced by Cummer and Inan, 2000 and used by Mackay, C.E.J et al., 2010 which shows an improvement in lightning distance estimation. However this improvement is also limited by assumptions made regarding the source and current of the lightning discharge.

On the other note, Ogawa has found that by examining the background noise in 1 $\mathrm{Hz}$ to $11 \mathrm{kHz}$, it is possible to observe a secondary waveform caused by the same lightning strikes. The secondary (antipodal) waveform results from the sferic propagating along 
the opposite direction around the world. With data from the direct and secondary waveforms, lightning location can be estimated (Ogawa et al., 2007), though in many cases it is hard identify the secondary waveforms . To overcome these problems, we introduce a lightning detection method based on observations from a single station that do not suffer from these limitations.

\section{Evolution of sferics time domain structure with distance}

Before introducing our method, we begin by inspecting some recorded sferics shown in Figure 2. By evaluating the data in this figure, we can make several key observations

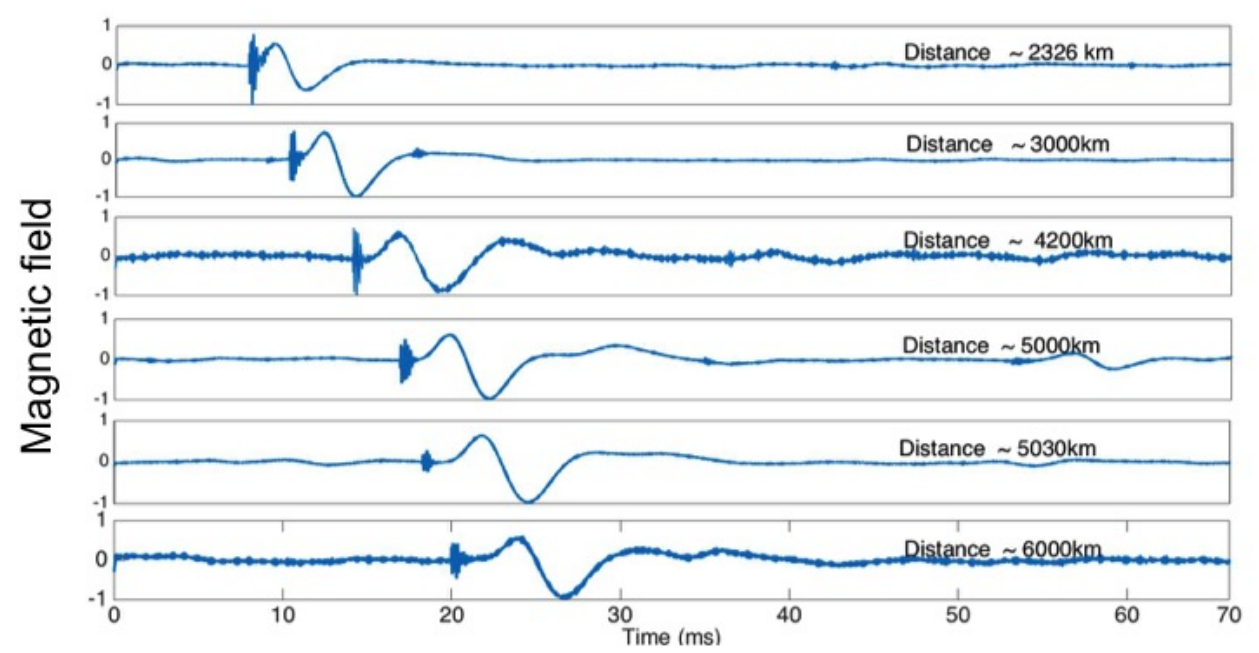

Figure 2. Example of sferics over different distances recorded during the night. Data recorded at Sondrestrom station

that will form the basis of our proposed algorithm. Each row in the figure corresponds to a lightning discharge reported by the NLDN dataset. We also used this dataset to calculate the propagation distance and to determine whether the sferic propagated during the daytime or nighttime. All of the sferics shown in Figure 2 propagated during the night. The zero point for each row is an NLDN time estimate for a lightning strike. Although the sferic magnitude is generally attenuated over propagation distance, here the magnitudes have been normalized. Therefore, the magnitude attenuation is not shown in Figure 2. This normalization will help us to see the patterns and variations in the waveform structure for different propagation distances, which is a key for our method. There are two key observations to make: as the propagation distances increase, 1) the time interval be- 
tween the zero time and the sferic start point increases, and 2) the separation time between the VLF component (initial burst) and the ELF component (subsequent component) also increases. In the following sections, we will show that these two time values are both proportional to propagation distance. This, along with phase velocity derivations described in section 4 and 5, will be the basis of our method to estimate the propagation distances.

\section{Galejs's model for the Earth-ionosphere wave guide}

In this work we use phase velocities derived from the Earth-ionosphere model developed by Galejs. Galejs formulated a model to study wave propagation in the spherical shell between the Earth and ionosphere (Galejs,J, 1972). The model makes a number of simplifying assumptions. It assumes the Earth and ionosphere boundaries are concentric spheres forming a spherical waveguide. The ionosphere is assumed to be a sharply bounded and homogeneous ionized medium. Galejs further assumes the height and conductivity of the ionosphere remain constant through the propagation path. Figure 3 illustrates this model with two boundaries located at $r=a$ and $r=a+h$, where $a$ is Earth's radius and $h$ is the ionosphere height. The source as a vertical dipole is located on the ground at $\theta=0, r=a+Z_{s}$, and the receiving antenna (shown as orthogonal loops) is located on the ground at $\theta=\theta_{0}, r=a+Z_{o}$. Since the ionization level of the ionosphere

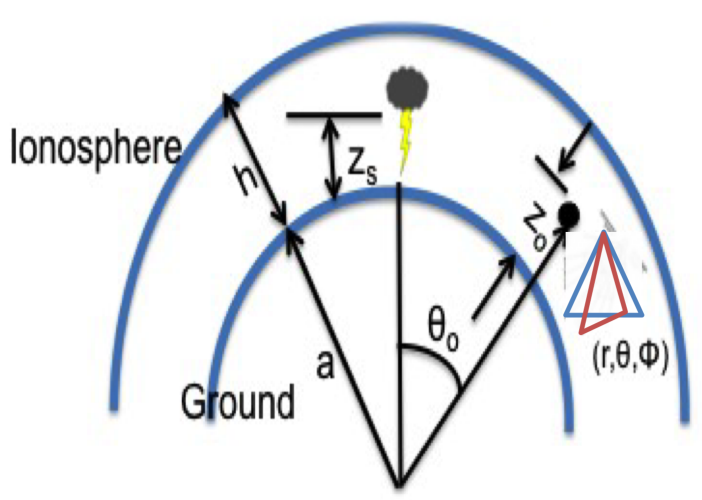

Figure 3. Geometry of the Earth-ionsphere spherical shell

is different in day and night, the height and conductivity of the ionosphere vary in daytime and nighttime (Wait, J. R., 1960). Considering this fact, in this paper and in the Galejs model, different values are assumed for the height and conductivity of the ionosphere depending on whether sferics propagate during day or night. During the day $h$ is set to 70 
$\mathrm{km}$ and the conductivity of the ionosphere is assumed to be $10^{-6} \mathrm{~S} / \mathrm{m}$. At night $h$ is set to $85 \mathrm{~km}$ and the conductivity of the ionosphere is assumed to be $10^{-5} \mathrm{~S} / \mathrm{m}$. The ground's conductivity is assumed $10^{-3} \mathrm{~S} / \mathrm{m}$ for day and night. The solution of radial fields observed at $r=a+Z_{o}$ is expressed in terms of spherical Bessel functions as :

$$
R(r)=r\left[A h_{\nu}^{(1)}(u)+B h_{\nu}^{(2)}(u)\right]
$$

where:

$$
h_{v}^{m}(u)=\left(\frac{\pi}{2 u}\right)^{0.5} H_{v+0.5}^{m}(u)
$$

and $H_{(v+0.5)}^{m}(u)$ is the Hankel function of kind $m$ and order $v+0.5, u=k_{0} r$ and $k_{0}$ is the wave number and is equal to

$$
k_{0}=\omega \sqrt{\mu_{0} \epsilon_{0}}
$$

$\mu_{0}$ and $\epsilon_{0}$ are the permeability and permittivity of free space, respectively. Due to lack of simple representations for the spherical Bessel functions of large order $v$ and $u$, a solution for approximation for thin shell was developed, where $\frac{h}{r} \rightarrow 0$. The factor $r$ is also approximated by the average value in the shell $\left(r_{m}=a+0.5 h\right)$. With this assumption, equation (1) reduces to equation (4) as below:

$$
R=A \exp (i K r)+B \exp (-i K r)
$$

where

$$
K^{2}=k_{0}^{2}\left[1-\left(a S / r_{m}^{2}\right)\right]
$$

and $S$ is the propagation parameter, which can be interpreted as the ratio between the wave number along the surface of the earth $k_{\theta}$ and the wave number of the free space $k_{0}$. In this paper, $S$ is used to find the velocity of both the VLF and ELF components of sferics. Galejs defined S using modal equation of T.M. modes for the VLF range in equation (6):

$$
S=\frac{r_{m}}{a} \sqrt{1-\left[\frac{(n-0.5) \pi}{k_{0} h}\right]^{2}}
$$

where $\mathrm{n}$ is T.M. mode number and can be one or an arbitrary integer. Galejs shows equation (6) is strictly valid for a conducting ground surface and a perfectly reflective ionosphere. However, these assumptions may idealize the boundaries in the VLF range and the results may slightly diverge from observations. In the ELF range, where only the TEM mode propagates, the waveguide boundaries appear as nearly perfect conductors and equation (6) can be reduced to equation (7):

$$
S=\frac{r_{m}}{a} \sqrt{1+\frac{i\left(\Delta_{e}+\Delta_{g}\right)}{k_{0} h}}
$$


where

$$
\Delta_{e}=\sqrt{\frac{\omega \epsilon_{0}}{\omega \epsilon_{0} \epsilon_{i}+i \sigma_{i}}}
$$

and

$$
\Delta_{g}=\sqrt{\frac{\omega \epsilon_{0}}{\omega \epsilon_{0} \epsilon_{g}+i \sigma_{g}}}
$$

$\Delta_{e}$ and $\Delta_{g}$ represent the normalized impedances for the ionosphere and ground, respectively. $\epsilon_{i}, \epsilon_{g}$ and $\epsilon_{0}$ are the permittivity of the ionosphere, ground, and free space, respectively. $\sigma_{i}$ and $\sigma_{g}$ are the electrical conductivity of the ionosphere and ground, respectively. Having defined $S$, Galejs went on to derive the phase velocities. In our method, the phase velocities are essential parameters for estimating lightning distances. In the next section we present the phase velocities for the VLF and ELF components of sferics using Galejs's model.

\section{Modeling waves 's phase velocities}

The phase velocity is inversely related to the real part of the propagation parameter as follows:

$$
V_{p h}=\frac{c}{\operatorname{Real}(s)}
$$

where $C=\frac{1}{\sqrt{\mu_{0} \epsilon_{0}}}$ is the free-space velocity of electromagnetic waves. Since the Galejs model is most accurate at lower frequencies, where the height of the waveguide is smaller than the wavelength (Galejs, J. et al., 1972), we can confidently apply this model to obtain the propagation parameter for low frequency bands. Figures 4 and 5 show $S$ in ELF and VLF frequencies, respectively. Later we use these graphs along with equation (10) to find the phase velocity for these components of the sferic. Using equation (7) we calculated the real part of propagation parameter in the ELF frequency band for both daytime and nighttime, which are shown in Figure 4. It follows from equation (7) and equation (3) that for an increase in frequency, $S$ will decrease. Figure 4 shows this as a downward trend in $\mathrm{S}$ over frequency. The first cut off frequency for the Earth-ionosphere waveguide is 1580 $\mathrm{Hz}$ (Inan, et al., 2000). As shown in Figure 4, at frequencies less $1580 \mathrm{~Hz}, S$ is greater than one. Since $\mathrm{S}$ is greater than one, according to Equation (10), the phase velocity in the ELF range is less than $c$. However, as the waveguide boundaries become perfect conductors the phase velocity of the ELF waves becomes nearly equal to $c$. This is because, in perfect conductors, normalized impedances $\left(\Delta_{e}\right.$ and $\left.\Delta_{g}\right)$ approach zero, causing $S$ to approach one. Figure 5 shows the propagation parameter of the VLF waves, calculated using Equation (6), with different values for daytime and nighttime.The value reported in 


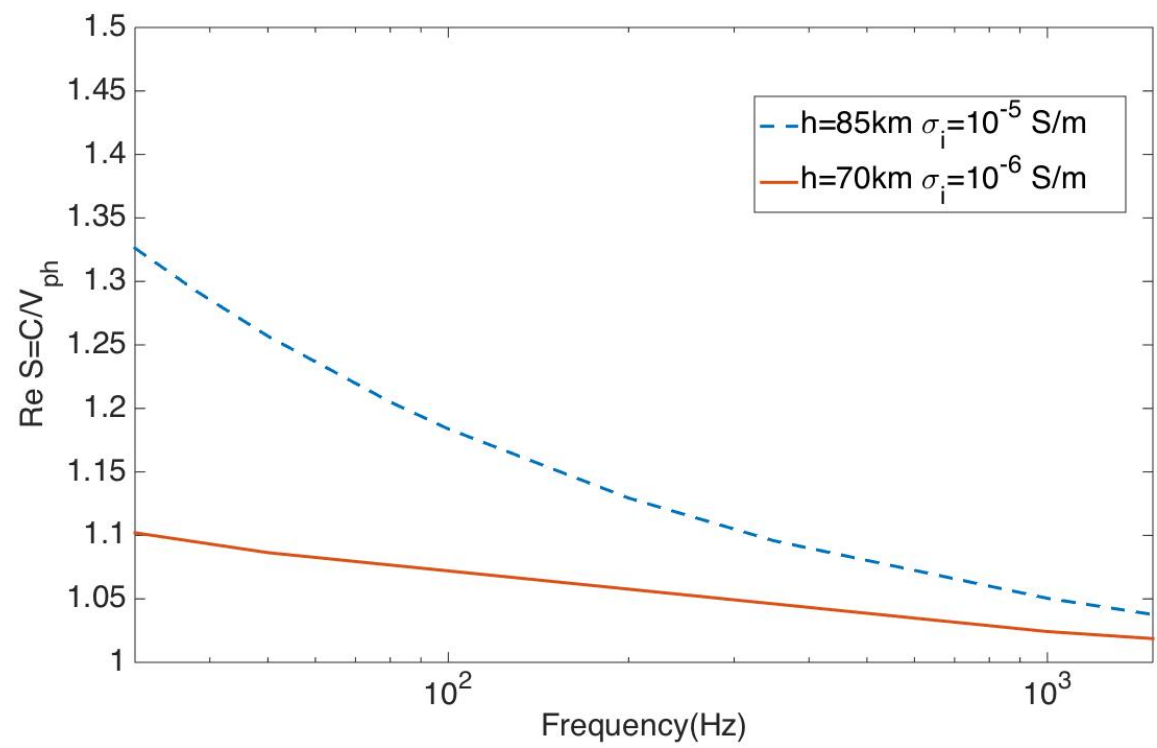

Figure 4. Propagation parameter of the ELF waves, red for day and blue for night

this graph is consistent with phase values reported by Wait, J. R., 1970. In contrast with the ELF range, the propagation parameter in the VLF range shows different behavior. As shown in Equation (6), an increase in frequency will result in an increase in $S$. This accounts for the upward trend observed in Figure 5.

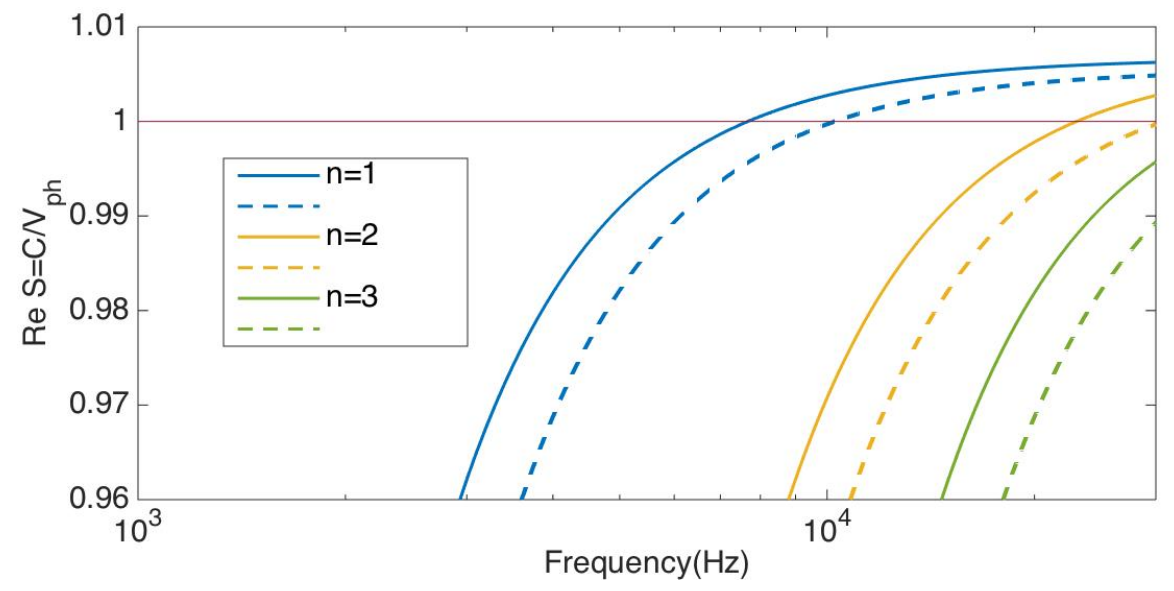

Figure 5. Propagation parameter of the VLF waves in the first three modes, dashed line for day and solid line for night 
One interesting observation from Equation (6): $S$ is imaginary for lower frequencies and, as a result, the waves in that frequency range are severely attenuated. But as the frequency approaches the cutoff frequencies, $k_{0}=k_{c}=\frac{(n-0.5) \pi}{h}$ and $S$ approaches zero. Thus, according to Equation (10), the phase velocity will be infinite at the cutoff frequencies. In the frequencies higher than the cutoff frequencies, $S$ will gradually becomes greater than one, and when that occurs, the phase velocity becomes less than $c$.

\section{Methodology}

Section 5 described a model how to obtain the phase velocities for the VLF and ELF components of the sferic. These values will be used as inputs in our method to estimate lightning distance. Our methodology can be divided into three main steps: 1) Sferic identifications in the recorded data 2) Distance and time estimation of lightning using ELF and VLF components of the identified sferic 3) Direction estimation. Finally, we employ all three of these steps to geolocate the lightning. Each of these steps are described in the following sections.

\subsection{Sferic identification in recorded data}

As mentioned before, the method presented here to find the lightning emission time and location is based on characteristics of the associated ELF and VLF components of sferics. Prior to using sferics, the ELF and VLF components must first be identified and isolated in the recorded data. An example of this isolation process is shown in Figure 6. Figure (6a) illustrates a typical sferic waveform generated by lightning in approximately $300 \mathrm{~Hz}-40 \mathrm{kHz}$ frequency range. Figures (6b) and (6c) show the isolated VLF and ELF components of the same sferic, after passing it through high- and low-pass filters, respectively. We also eliminated the phase shifts introduced by filters by compensating the group delays. The waveform shown above was received by a NS directed antenna . However, the closer the sferic 's arrival direction is to the EW direction, the less the NS antenna will be able to capture the signal. As a result, to capture a sferic coming from any possible direction, we need to consider recorded data from the antenna directed in EW, the orthogonal direction, as well. Figure (7a) shows the sferic displayed in Figure (6) but now includes both NS and EW directions, shown in red and blue, respectively. To identify a sferic's waveform coming from any direction, we calculate the envelope of the signal $\left(N S^{2}+E W^{2}\right)^{0.5}$ from the recorded data, shown in Figure (7b). To determine the arrival 


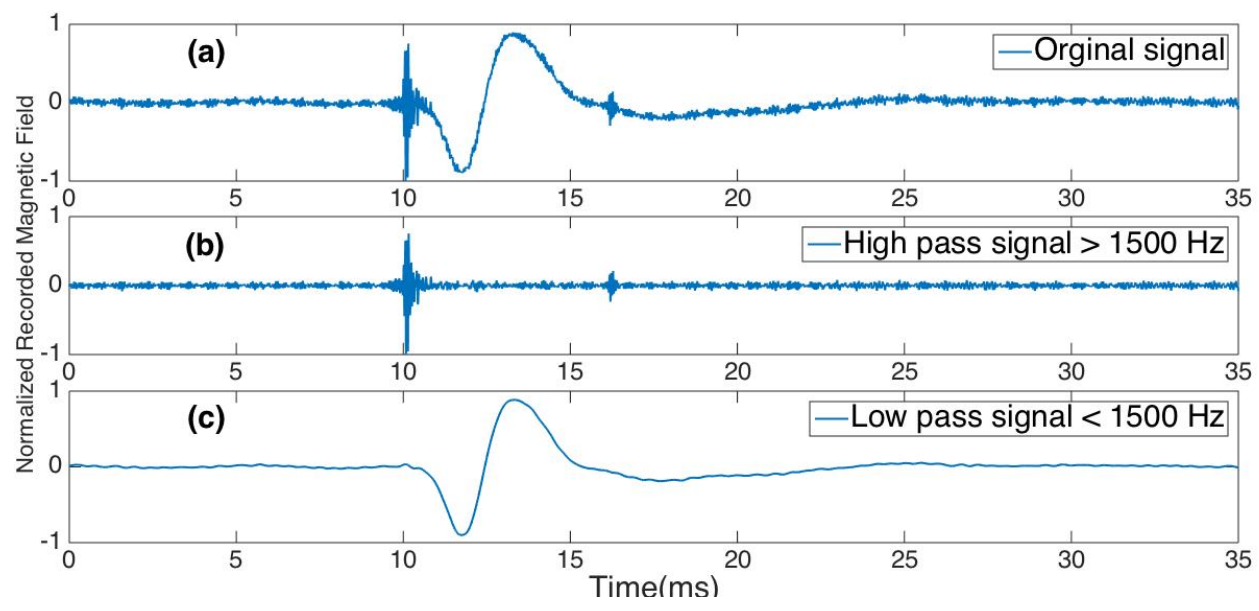

Figure 6. A typical sferic waveform recorded in the Arrival Height station (a), the high-pass waveform (>1500 Hz; VLF component) (b), and the low-pass waveform( $<1500 \mathrm{~Hz}$; ELF component) (c).
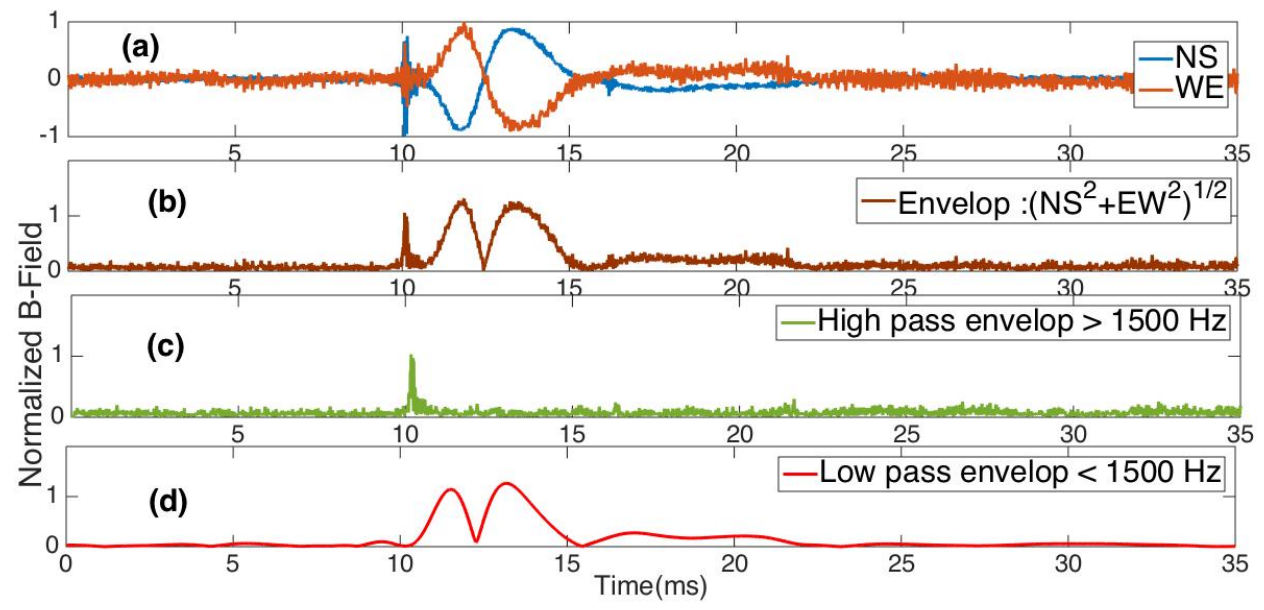

Figure 7. The same recorded sferic as in Figure 6 now including NS and EW directions (a), envelope in $300 \mathrm{~Hz}-40 \mathrm{kHz}$ band (b), high-pass envelope (>1500 Hz; VLF component) (c), and the low -pass envelope (<1500 Hz; ELF component) (d).

time of the VLF component $\left(t_{V L F}\right)$, we passed the envelope signal through a high pass filter with a cutoff frequency of $1500 \mathrm{~Hz}$. The high- pass envelope of the corresponding sferic is shown in Figure (7c). Using the high-pass envelope, we determine $t_{V L F}$ to be the global maximum point in that envelope. To find the arrival time of the ELF component $\left(t_{E L F}\right)$, we passed the envelope signal through a low pass filter using the same cutoff frequency as the VLF component. The low pass envelope of the corresponding sferic 
is shown in Figure (7d). We also defined the first global peak as the arrival time of the sferic.

It should be noted that the detection efficiency of proposed distance estimation method is limited to the percentage of sferics with clear ELF components. There exists a number of factors that limits the percentage of clear sferics. One of these factors is the interference with other recorded sferics which reduces the percentage of clear and usable sferics. Mackay C.E.J, et al., 2012 shows that on average, 70 percent of the recorded sferics are interference-free. Another limiting factor is noise threshold. For a sferic to be detectable in a particular station, the sferic power must be higher than the noise level threshold in that station. It means the magnitude of peak current of the sferic should be greater than the station noise cutoff and the attenuation of propagation path.

\subsection{Distance Estimation algorithm}

All of the previous sections have provided us with required inputs for a novel algorithm that is described in the following sections. To start, assume that the lightning discharge happened at $t_{\text {initial }}$ at $d$ distance from our receiver. And also assume $t_{V L F}$ and $t_{E L F}$ are the arrival times of the VLF and ELF components, respectively. Also assume that the waves in ELF and VLF bands propagate along the same path to reach the antennas. Given these parameters and according to the time-velocity relationship:

$$
\begin{aligned}
& V_{V L F}=\frac{d}{t_{V L F}-t_{\text {initial }}} \\
& V_{E L F}=\frac{d}{t_{E L F}-t_{\text {initial }}}
\end{aligned}
$$

Solving Equation (11) and (12) leads to:

$$
\begin{gathered}
t_{\text {initial }}=\frac{t_{V L F} V_{V L F}-t_{E L F} V_{E L F}}{V_{V L F}-V_{E L F}} \\
d=a\left(t_{E L F}-t_{V L F}\right)
\end{gathered}
$$

Where $a$ is a constant and

$$
a=\frac{V_{E L F} V_{V L F}}{V_{V L F}-V_{E L F}}
$$

In equation (14), $t_{E L F}-t_{V L F}$ is the separation time between the arrival of waves in the ELF and the VLF bands and can be calculated from the waveform's characteristics as described in section 6-1. As deduced from equation (13) and equation (14), we can see that 
only the velocity and arrival times for the ELF and VLF components from a single station are necessary to find emission time and the propagation distance of the lightning strikes. This frees us from the traditional locating methods, which required recording data from multiple stations. Since the profile of the ionosphere differs during daytime and night time, characteristics of a sferic also varies depending on whether it propagated during the day, at night, or across the day-night terminator. The proposed distance estimation method assumes the sferic propagated during the day or at night. When the sferic crosses the daynight terminator, signal propagation should be modeled over nonuniform waveguide which leads to a much more complex problem. As a possible solution, propagation parameters can be averaged to determine attenuation rates and phase velocities of sferics crossing the day-night terminator. A more complex solution involves modeling attenuation as a dependent of propagation distance and time of day (Mackay, C.E.J et al(2010)).

\subsection{Additional Distance Estimation method}

In certain circumstances, there is an additional method that can be used along with our method. When these two methods are used together, they yield more accurate distance approximations from recorded data. Ogawa found that with a strong lightning strike it is possible to observe a secondary waveform caused by the same lightning strike (Ogawa, et al., 2007). These antipodal waves propagate in the opposite direction across the globe (Figure 8). The difference in arrival times between the antipodal wave and direct wave can also be used to estimate the distance between antennas and individual lighting occurrences.

Figure 8 shows an ELF sferic generated by a single lightning return stroke, which has three noticeable components. The first burst is correlated to the shortest path from the lightning to the receiver; antipodal path generate the second burst and the third burst is the shortest path and a delay from one around-the-world (ATW) path. Since the antipodal wave needs to travel at least half of Earth's circumference ( $\sim 20 \mathrm{Mm})$, the VLF component of the lightning will be significantly attenuated. However, the ELF component will not display the same level of attenuation and, because of that it should generally be visible in the spectrum data radiated by antipodal waves. Considering this fact, the time -velocity relationship for the direct and antipodal waves are shown in equation (16) and (17): 

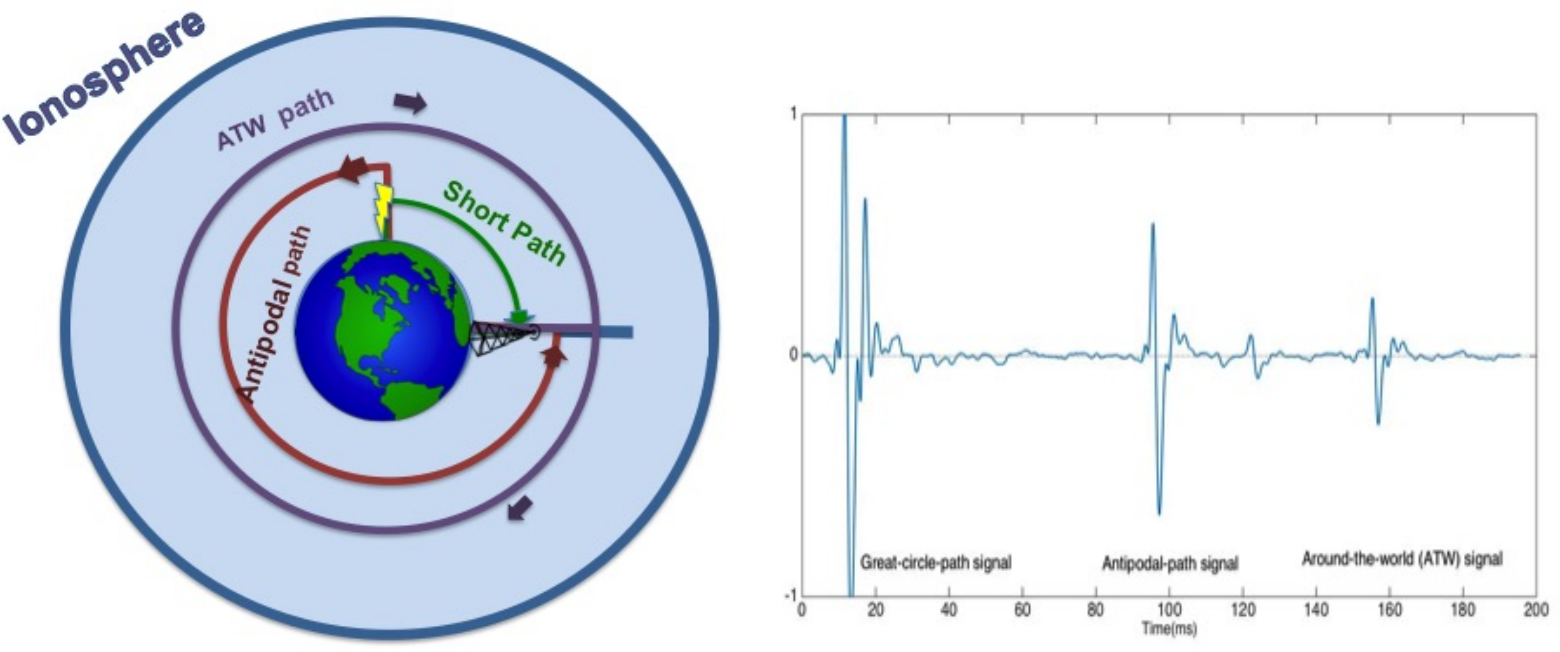

Figure 8. The illustrations of Short path and antipodal path (a), a particular (ELF) sferic waveform with three noticeable bursts recorded in the Arrival Height station (b)

$$
V_{E L F}=\frac{d}{t_{\text {Direct }}-t_{\text {initial }}}
$$

$$
V_{E L F}=\frac{40 M-d}{t_{\text {Antipodal }}-t_{\text {initial }}}
$$

Where $V_{E L F}$ is the phase velocity of waves in ELF range $d$ is the shortest distance between return stroke and receiver antenna. $t_{\text {Direct }}$ and $t_{\text {Antipodal }}$ are correlated to direct and antipodal waves respectively. Solving equations (16) and (17) for $d$ leads to equation (18):

$$
d=20 M m-\frac{V_{E L F}}{2}\left(t_{\text {Antipodal }}-t_{E L F}\right)
$$

In conclusion, since both Equation (14) and (18) estimate propagation distance separately, using both equations and averaging their distance estimations can potentially lead to a more accurate result. While the Ogawa method is also a single station method for estimating the propagation distances, it has some restrictions that limit it uses when compared to our method. In particular it is limited to cases where lightning strikes are strong enough that attenuated ELF waves are still visible in the recorded data. Another limitation is that this method is restricted to cases where the lightning occurred not too close to the receiver, because in this case the antipodal wave will be highly attenuated and not visible in the received data. The other disadvantage of Ogawa method is that in his equation, the 
velocity of the waves stays the same for the whole propagation path. However, the antipodal waves must cross the day-night terminator, which requires considering different wave velocities for day and night.

In comparison to single station method presented in Ogawa. et al (2007), the proposed method described in section 6.2 does not require observing antipodal peak on the sferic's structure, making it more widely applicable. Also compared to ( Mackay C.E.J, et al., 2012), another single station method, this method is less resource intensive and requires less processing cycles and memory usage.

\subsection{Direction estimation}

Given the estimated distance between lightning strike and receiver, to obtain the location of the lightning we only need to find the sferic's incoming direction. Horizontal magnetic field measurements could be used in the magnetic direction finding(MDF) of lightning discharges (Füllekrug, $M$, 2017). To do this, we use MDF method for the single station which was introduced by Said, R. K. et al., 2010. This method also uses data from two orthogonal loop antennas that are positioned in NS and EW directions. The incoming sferic excites the NS and EW antenna, and as shown in Figure 9, inducing signal $B_{1}(t)$ and $B_{2}(t)$ in NS and EW antennas respectively Since the ratio of $B_{1}(t)$ and $B_{2}(t)$ is

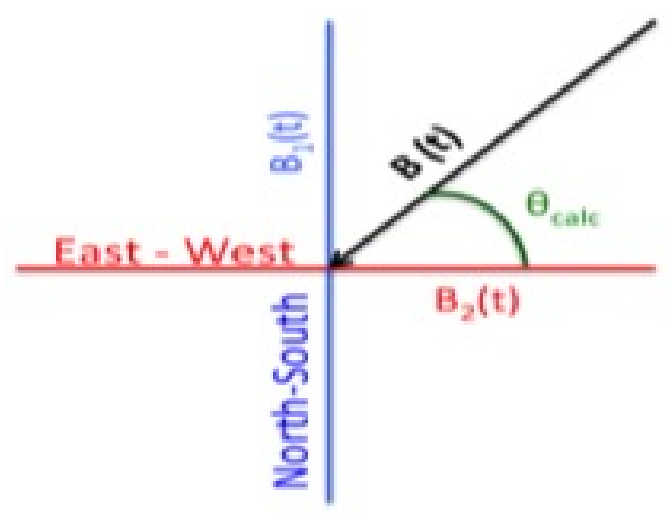

Figure 9. An illustration for finding the direction of lightning

proportional to the tangent of the azimuth, they can be sampled and used to find the incoming direction of the sferic. Figure 10 illustrates an implementation of Said, R. K. et al.'s, 2010 method using our data. As the method is developed for the VLF component of the sferic, we first isolated the VLF component by passing it through a high pass fil- 

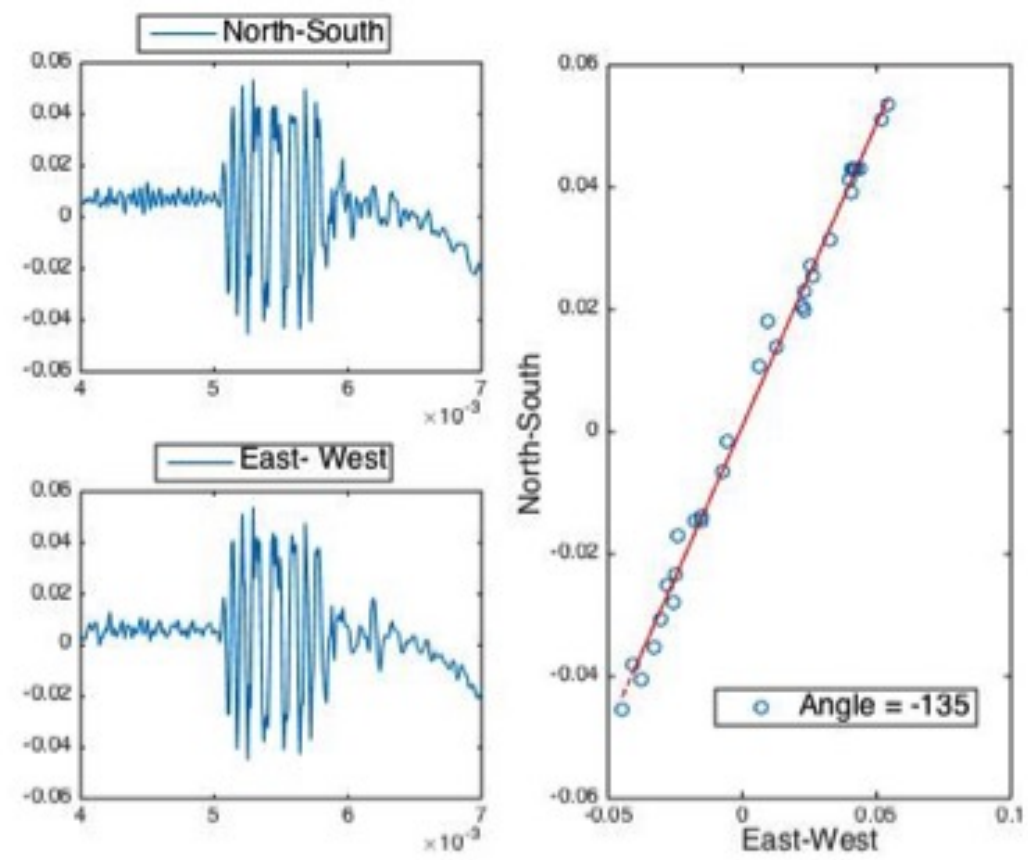

Figure 10. An example of direction finding algorithm. North-South and East-West measurements are plotted versus time in left-hand figures. The right hand figure display North-South vs East-West recording and the best linear fitting in solid line. The azimuth of the sferics calculated as $\theta_{\text {calc }}=45$.

ter $(1500 \mathrm{~Hz})$. After isolation, we plotted the NS recording against the EW recording for the first $500 \mu \mathrm{s}$. The tangent of the azimuth is the slope of the least-squared fitting line of the plot. However, if the data recorded from one of the antennas is noisy the accuracy of this method will dramatically drop. We found out that an improvement can be achieved in direction estimation if we instead consider sferic waveform in full bandwidth and not limit ourselves to only the VLF part of the sferics. This would add more data points to the model and potentially helps fitting a more accurate line. Figure 11 illustrates an our improvement on Said's method on a noisy recorded data. One potential limitation to this method could be the cases where the ELF component has a lower SNR than the VLF component, where considering noisy ELF data, could drop the accuracy of fitting line. Also since the crossed antennas at the site are never perfectly aligned to the geographical North-South and East-West, we used equation (19) to compute a corrected arrival azimuth, $\theta_{\text {corr }}$, using the calculated azimuth, $\theta_{\text {calc }}$, and the correction factors specific to the recording site.

$$
\theta_{\text {corr }}=\tan ^{-1}\left[\alpha \frac{\tan \left(\theta_{\text {calc }}\right)}{\cos \xi}-\tan \xi\right]+\phi
$$



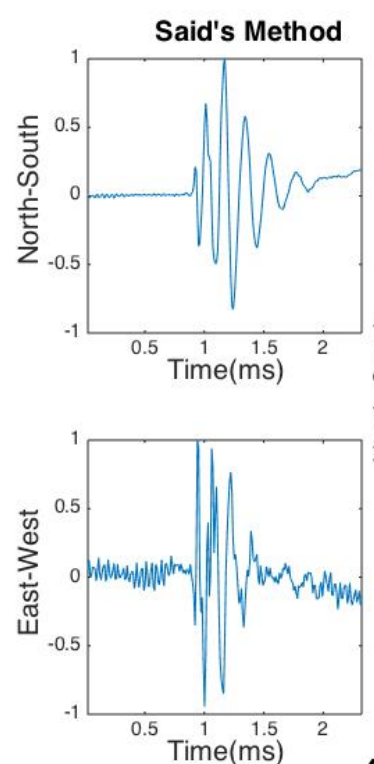

(a)

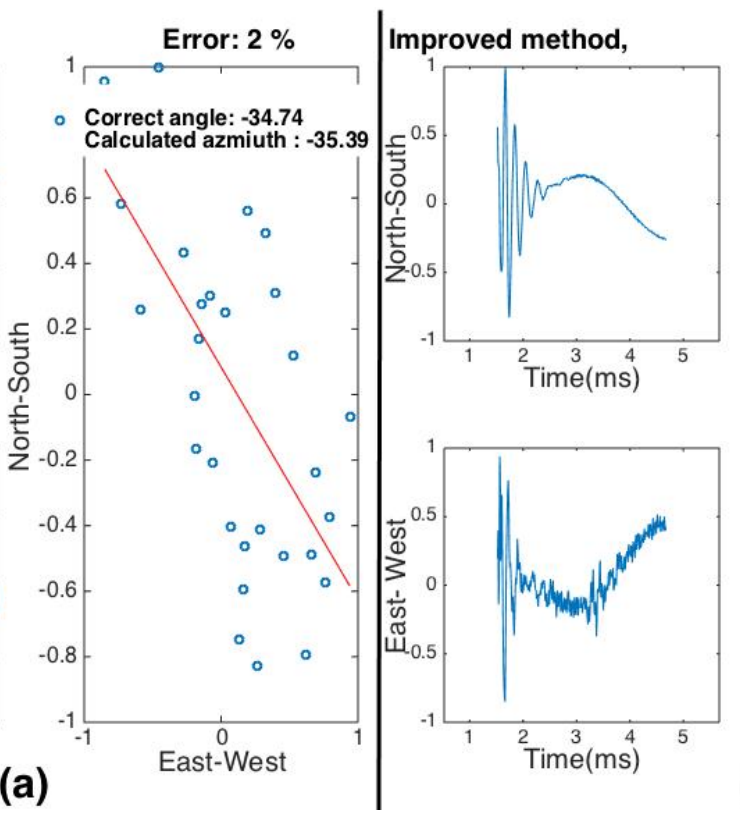

Figure 11. Direction approximation for a sample sferic recorded in Sondrestrom using Said,R. et al., 2010 method (a), using proposed MDF method (b)

For results presented in this paper, which are recorded at Sondrestrom, correction factors are as follow: $\alpha=1, \xi=20^{\circ}$ and $\phi=79^{\circ}$ (Mackay, C.E.J et al., 2012).

\section{Results}

To estimate the distance of propagation, we employed our proposed method (described by equation (14)) along with the Ogawa method (described by equation (17)) when possible. By using both equations and averaging their distance estimations, we can potentially gain a more accurate result. However, the Ogawa method could only be applied to a limited number of sferics where special conditions mentioned in section 6-3 are satisfied. Method correctness requires that the velocity computations and arrival-time measurements be computed in the same frequency, since both are frequency-dependent. In this work, we have arbitrarily selected $500 \mathrm{~Hz}$ and $30 \mathrm{kHz}$ as operating frequencies for the ELF and VLF computations, respectively. To use our proposed method we need to find the constant $a$ (equation (14)). This constant is a function of the ELF and VLF velocities, which are themselves a function of their respective propagation parameters. For the ELF component, we applied f $=500 \mathrm{~Hz}, \sigma_{i}=10^{-5} \mathrm{~S} / \mathrm{m}$, and we obtained $S=1.05$ for 
the nighttime profile. Substituting the $S$ value in equation (10) gives a phase velocity of $\sim 2.85 \times 10^{8} \mathrm{~m} / \mathrm{s}$.

For the VLF component, we used equation(6) where $\mathrm{f}=30 \mathrm{kHz}, \sigma_{i}=10^{-5} \mathrm{~S} / \mathrm{m}$, and we obtained $S=1.005$ for the night. Substituting the $S$ value in equation (6), gives a phase velocity around $2.98 \times 10^{8} \mathrm{~m} / \mathrm{s}$ for the night.

Substituting the above phase velocity values in equation (14) gives distance estimations described below in equation (20).

$$
d(\text { night })=6.66 \times 10^{9}\left(t_{E L F}-t_{V L F}\right)
$$

Note that equation (20) only applies for sferics in which height of the ionosphere is constant in their propagation path, i.e., those sferics that do not cross the day-night terminator. Figures 12 shows a histogram of the estimated error for lightning distance for a set of 100 sferics occurred in night. As seen from Figures 12, distance estimation has an

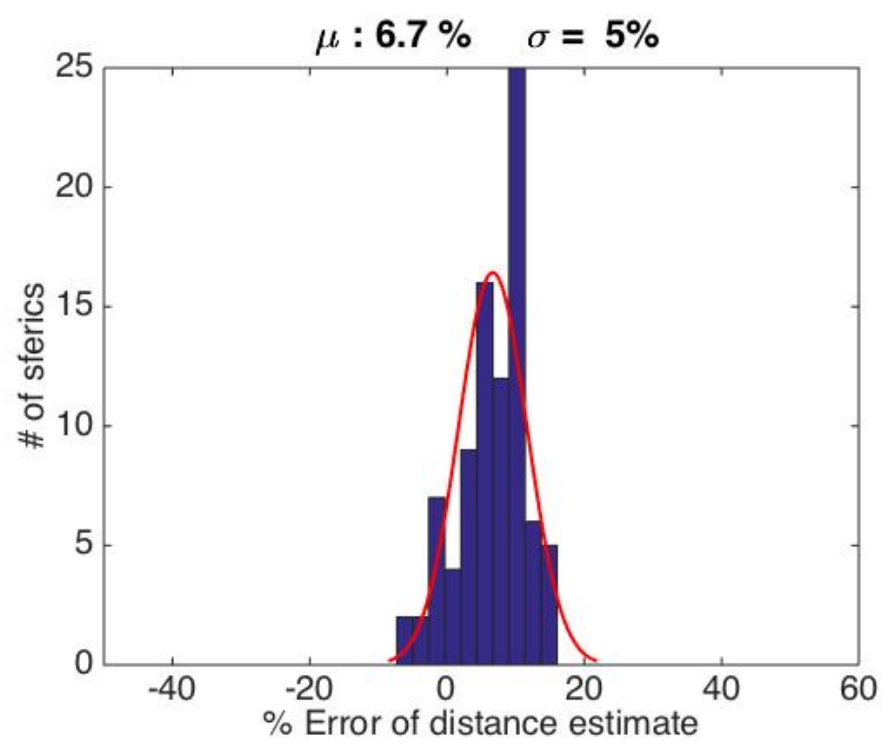

Figure 12. A histogram of the error values for distance approximation for 100 sferics

average error of $\sim 6.7 \%$ with $\sim 5 \%$ standard deviation. We further compared our time emission estimations with those of the NLDN for the same strikes. It is worth noting that this average error is in percentage of the actual distance between station and the lightning stroke and would increase as station-lightning stroke distance increases.

The histogram of the time emission approximation error is shown in Figure 13. The result shows $\sim 2 \times 10^{-4} \%$ average error and $\sim 3 \times 10^{-4} \%$ standard deviation. The coverage 


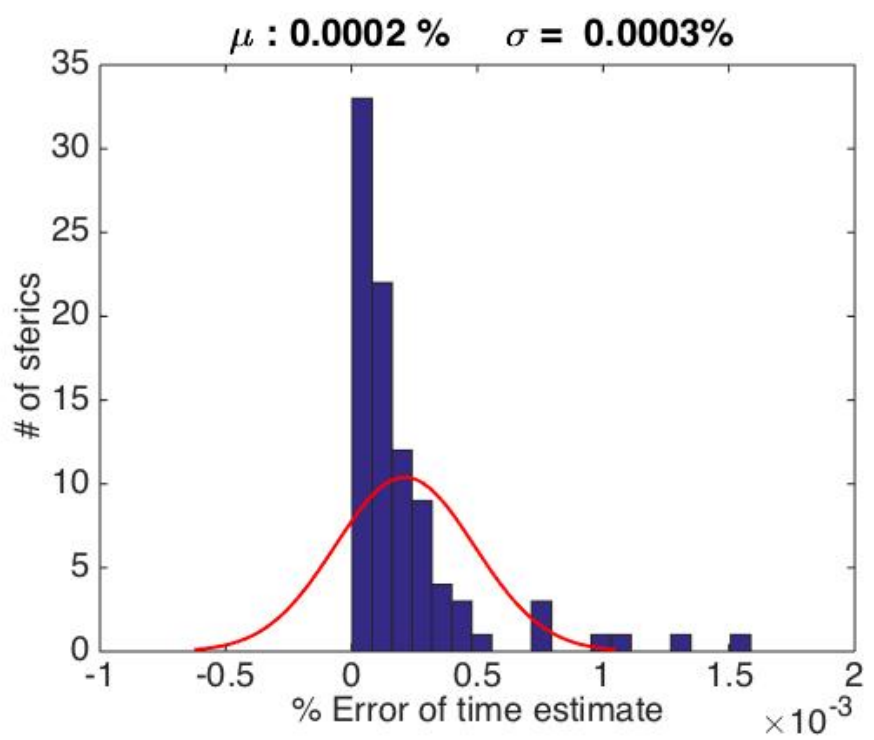

Figure 13. A histogram of error values for time estimation for 100 sferics

range of the method can be estimated using distribution of the lightning's peak currents and also minimum detectable peak current around the globe. Sondrestorm station mostly covers lightnings occurring in the Northern American and Western European regions ( Mackay C.E.J, et al., 2012). The error estimation results for the direction finding method described in section (6-4), are also shown in Figure 14a. This method yields average error $\sim 6.7 \%$ with $\sim 17.6 \%$ standard deviation. Using proposed improvements in section 6-4, we also computed a histogram of the error for direction approximation for same set of sferics. As seen in Figure 14b the average error dropped by $\sim 5.4 \%$ in comparison with the method proposed by Said, R. K. et al., 2010.

\section{Conclusion}

We have introduced a novel method to estimate the propagation distance and the emission time of sferics using recorded data from single station. Our method is more accurate and more widely applicable than the Ogawa method while being less resource intensive than other single-station methods (Mackay C.E.J, et al., 2012) as well as multistation methods, like the NLDN.

These improvements were achieved first by making key observations into the time relationship between ELF and VLF components of sferics. With these observations and the Galejs method for approximating the phase velocities we estimated the propagation 

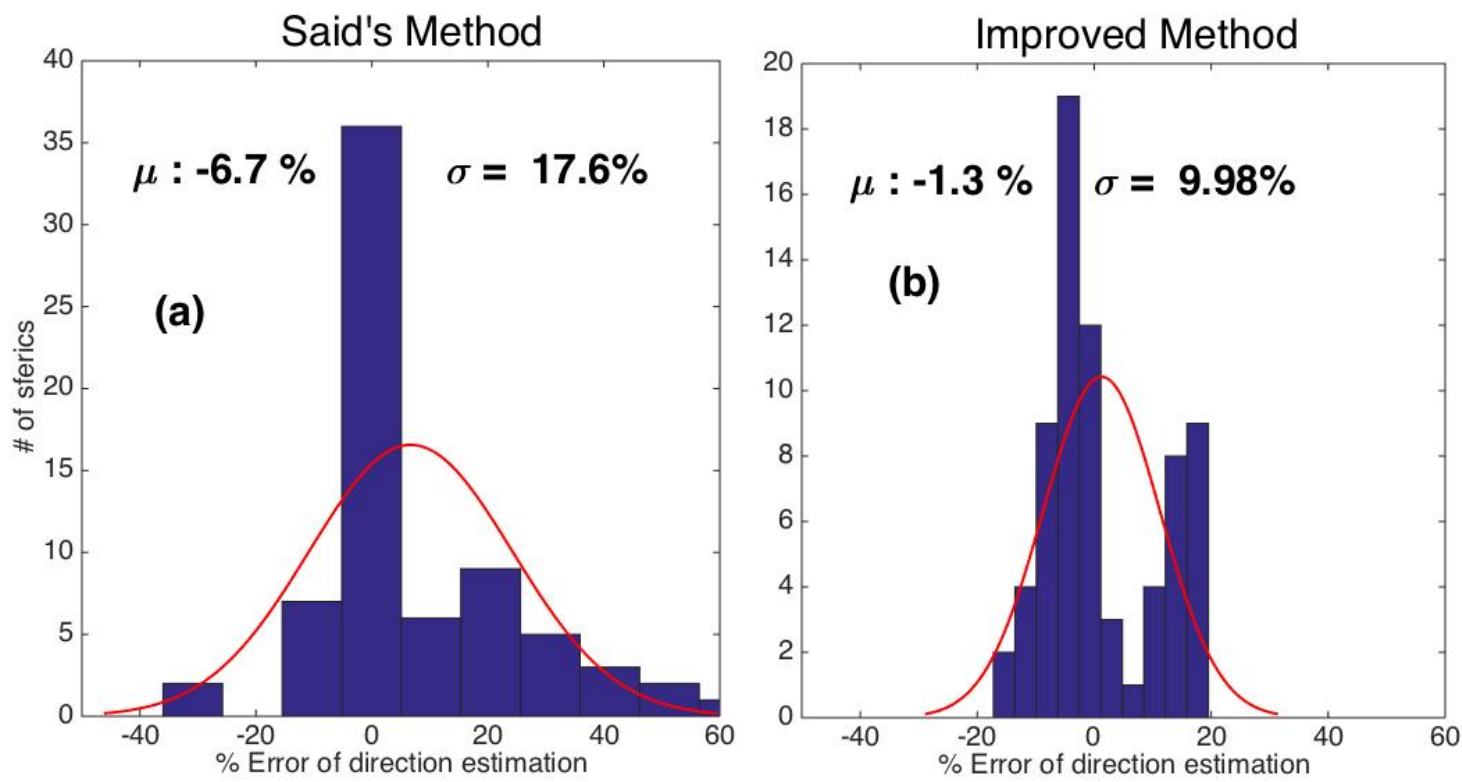

Figure 14. A histogram of error values for direction estimations for 100 sferics using Said,R. et al., 2010 (a), using modified MDF for single station (b)

distance of the sferics. Additional improvements were achieved by estimating the azimuth of the sferic by considering the whole sferic waveform and not just the VLF part.

In short, while our method may be less accurate than multiple station methods, it is more accurate and less resource intensive than previous single-station methods. Given the advantages of using single station measurements relative to using multiple-station methods such as reduced cost for building, maintenance, and synchronization of the stations, it is a great alternative to multiple- station methods in many applications where the precise location of individual lightning discharges isnot not the significant factor. Identifying lightning storm regions is the important factor where can be achieved using the proposed method by clustering the measurements of each single lightning within the storm.

\section{Acknowledgments}

Partial support for this research was provided by the Division of Polar Programs of the National Science Foundation through award 0944773 and by the Office of Naval Research through grant N00014-10-1-0378. The experimental data used to prepare figures are archived on site below:

https://stanfordmedicine.box.com/s/0mtcg8svm9v4o8ue4tajg7b2au6c5oba 


\section{References}

Burke, C. P., \& Jones, D. L. (1995). Global radiolocation in the lower ELF frequency band. Journal of Geophysical Research: Atmospheres, 100(D12), 26263-26271.

Cummins, K. L., \& Murphy, M. J. (2009). An overview of lightning locating systems: History, techniques, and data uses, with an in-depth look at the US NLDN. IEEE Transactions on Electromagnetic Compatibility, 51(3), 499-518.

Füllekrug, M.(2017), Introduction to lightning detection. Weather, 72(2), 32-35.

Galejs, J. (1972)). Terrestrial propagation of long electromagnetic waves: International series of monographs in electromagnetic waves, Pergamon, 1972, Pages 74-149

Hughes, H. G., and R. J. Gallenberger (1974), Propagation of extremely lowfrequency(ELF) atmospherics over a mixed day-night path, J. Atmos. Terr. Phys., 36 (10), 16431661, doi:DOI:10.1016/0021-9169(74)90202-5.

Inan, U. S., \& Inan, A. S. (2000). Electromagnetic waves

Mackay, C., \& Fraser-Smith, A. C. (2010). Lightning location using the slow tails of sferics. Radio Science, 45(5).

Mackay, C. E. J. (2012). Locating Lightning from Slow-tail Measurements Recorded at a Single Station (Doctoral dissertation, Stanford University).

Murphy, M. (2018). Locating lightning. Physics Today, 71(3), 32-38.

Nag, A., Mallick, S., Rakov, V. A., Howard, J. S., Biagi, C. J., Hill, J. D., ... \& DeCarlo, B. A. (2011). Evaluation of US National Lightning Detection Network performance characteristics using rocket-triggered lightning data acquired in 2004-2009. Journal of Geophysical Research: Atmospheres, 116(D2).

Nag, A., Murphy, M. J., Schulz, W., Cummins, K. L. (2015). Lightning locating systems: Insights on characteristics and validation techniques. Earth and Space Science, 2(4), 6593.

Ogawa, T., \& Komatsu, M. (2007). Analysis of Q burst waveforms. Radio Science, 42(2).

Price, C., Asfur, M., Lyons, W., \& Nelson, T. (2002). An improved ELF/VLF method for globally geolocating sprite-producing lightning. Geophysical Research Letters, 29(3).

Ramachandran, V., Prakash, J.N., Deo, A. and Kumar, S., 2007, July. Lightning stroke distance estimation from single station observation and validation with WWLLN data. In Annales Geophysicae (Vol. 25, No. 7, pp. 1509-1517). 
Said, R. K., Inan, U. S., \& Cummins, K. L. (2010). Long-range lightning geolocation using a VLF radio atmospheric waveform bank. Journal of Geophysical Research: Atmospheres, 115(D23).

Said, R. K., Cohen, M. B., \& Inan, U. S. (2013). Highly intense lightning over the oceans: Estimated peak currents from global GLD360 observations. Journal of Geophysical Research: Atmospheres, 118(13), 6905-6915.

Said, R.K., (2017). Towards a global lightning locating system. Weather, 72(2), 36-40.

Wait, J. R. (1960). Terrestrial propagation of very-low-frequency radio waves, a theoretical investigation. J. Res. Nat. Bureau Stand, 64, 153

Wait, J. R. (1960). On the theory of the slow-tail portion of atmospheric waveforms. Journal of Geophysical Research, 65(7), 1939-1946.

Wait, J. R. (1970). Electromagnetic Waves in Stratified Media,Pergamon. 